\title{
Three quasi-stellar objects acting as strong gravitational lenses ${ }^{\star}$
}

\author{
F. Courbin ${ }^{1}$, C. Faure $^{1}$, S. G. Djorgovski ${ }^{2,3}$, F. Rérat ${ }^{1}$, M. Tewes ${ }^{1}$, G. Meylan ${ }^{1}$, D. Stern ${ }^{4}$, \\ A. Mahabal ${ }^{2}$, T. Boroson ${ }^{5}$, R. Dheeraj ${ }^{6}$, and D. Sluse ${ }^{7,8}$ \\ ${ }^{1}$ Laboratoire d'astrophysique, École Polytechnique Fédérale de Lausanne (EPFL), Observatoire de Sauverny, 1290 Versoix, \\ Switzerland \\ e-mail: cecile.faure@epfl.ch \\ 2 Division of Physics, Mathematics, and Astronomy, California Institute of Technology, Pasadena, CA 91125, USA \\ 3 Distinguished Visiting Professor, King Abdulaziz University, 21589 Jeddah, Saudi Arabia \\ 4 Jet Propulsion Laboratory, California Institute of Technology, Mail Stop 169-221, Pasadena, CA 91109, USA \\ 5 National Optical Astronomy Observatory, Tucson, AZ 85719, USA \\ 6 The University of Maryland, College Park, MD 20742, USA \\ 7 Astronomisches Rechen-Institut am Zentrum für Astronomie der Universität Heidelberg, Mönchhofstrasse 12-14, \\ 69120 Heidelberg, Germany \\ 8 Argelander-Institut für Astronomie, Bonn Universität, Auf dem Hügel 71, 53121 Bonn, Germany
}

Received 5 September 2011 / Accepted 21 October 2011

\begin{abstract}
We report the discovery of three new cases of quasi-stellar objects (QSOs) acting as strong gravitational lenses on background emission line galaxies: SDSS J0827+5224 $\left(z_{\text {QSO }}=0.293, z_{\mathrm{s}}=0.412\right)$, SDSS J0919+2720 $\left(z_{\mathrm{QSO}}=0.209, z_{\mathrm{s}}=0.558\right)$, SDSS J1005+4016 $\left(z_{\mathrm{QSO}}=0.230, z_{\mathrm{s}}=0.441\right)$. The selection was carried out using a sample of 22,298 SDSS spectra displaying at least four emission lines at a redshift beyond that of the foreground QSO. The lensing nature is confirmed from Keck imaging and spectroscopy, as well as from HST/WFC3 imaging in the $F 475 W$ and $F 814 W$ filters. Two of the QSOs have face-on spiral host galaxies and the third is a QSO+galaxy pair. The velocity dispersion of the host galaxies, inferred from simple lens modeling, is between $\sigma=210$ and $285 \mathrm{~km} \mathrm{~s}^{-1}$, making these host galaxies comparable in mass with the SLACS sample of early-type strong lenses.
\end{abstract}

Key words. gravitational lensing: strong - quasars: individual: SDSS J0013+1523 - quasars: individual: SDSS J0827+5224 quasars: individual: SDSS J0919+2720 - quasars: individual: SDSS J1005+4016

\section{Introduction}

Strong gravitational lensing is now a standard tool to study the mass distribution in galaxies. About 250 cases of galaxies acting as strong lenses on a background object (quasar or galaxy) are known to date and have been observed with Hubble Space Telescope (HST) (Muñoz et al. 1998; Bolton et al. 2008; Faure et al. 2008). This growing sample of lenses allows us to measure the total radial mass profile of galaxies using the combined constraints given by the shape of the lensed source and the dynamics of the lensing galaxy (e.g., Auger et al. 2010; Barnabè et al. 2009) and to probe sub-structures in lensing galaxies (e.g., Vegetti et al. 2010; Chantry et al. 2010; Yoo et al. 2005; Biggs et al. 2004; Kochanek \& Dalal 2004; Keeton et al. 2003; Koopmans et al. 2002; Mao \& Schneider 1998). When the lensed source is photometrically variable, like a QSO, the time delay constraint can also be used (Refsdal 1964) to measure

* Based on data obtained at the W. M. Keck Observatory, which is operated as a scientific partnership among the California Institute of Technology, the University of California and the National Aeronautics and Space Administration. The Observatory was made possible by the generous financial support of the W. M. Keck Foundation. Also based on observations made with the NASA/ESA Hubble Space Telescope, obtained at the Space Telescope Science Institute, which is operated by the Association of Universities for Research in Astronomy, Inc., under NASA contract NAS 5-26555. These observations are associated with program \#GO12233. the Hubble parameter, $H_{0}$, independently of any standard candle (e.g., Suyu et al. 2010).

Recently, we started building a new sample of lenses where the lensing object is a QSO host galaxy. Our goal is to weigh QSO host galaxies for the first time with strong gravitational lensing and to compare their dynamical and lensing masses. We presented the search method in Courbin et al. (2010) which included the first case of a QSO acting as a strong lens, based on Keck adaptive optics (AO) imaging observations. In the present paper, we show three new strong "QSO lenses" for which we have Keck optical imaging and spectroscopy as well as deep HST images in two bands with the WFC3 camera in the visible channel. Our sample, when completed, will consist in a tool to test directly the scaling laws established between the properties of quasars emission lines, the mass of the central black hole, and the total mass of the host galaxies (e.g., Kaspi et al. 2005; Bonning et al. 2005; Shen et al. 2008).

Throughout this paper, the WMAP5 $\Lambda$ CDM cosmology is assumed $\left(\Omega_{\mathrm{m}}=0.258, \Omega_{\Lambda}=0.742, H_{0}=72 \mathrm{~km} \mathrm{~s}^{-1} \mathrm{Mpc}^{-1}\right)$. All magnitudes are in the AB system.

\section{Object selection in SDSS}

Our search technique is similar to the one used to build the SLACS sample (Bolton et al. 2008) and was originally proposed by Warren et al. (1996). It consists of selecting lenses following their spectroscopic properties and looking for emission lines 
redshifted beyond the redshift of the potential lens. SLACS selects early-type massive galaxies as lenses. We select QSOs.

We use the SDSS DR7 catalogue (Abazajian et al. 2009) of spectroscopically confirmed QSOs to build a sample of potential lenses, without applying any magnitude cut. We consider all 22298 QSOs with $z_{\text {QSO }}<0.7$ and with SDSS spectra. In these spectra, we look for emission lines redshifted beyond the redshift of the QSO. Since the SDSS fiber is comparable in size ( $3^{\prime \prime}$ in diameter) to the typical Einstein radius of our QSOs, the object responsible for the background emission lines is very likely to be strongly lensed (e.g., Bolton et al. 2008).

The search technique itself consists in cross-correlating the QSO spectra with templates of emission line galaxies. The crosscorrelation is carried out after removal of the QSO continuum. We choose very simple templates, with no continuum but which contain the optical [O II] doublet, the $\mathrm{H} \beta$ hydrogen line, the [O III] doublet, and the $\mathrm{H} \alpha$ line. The best candidates selected by this technique, applied to SDSS spectra, show four clear emission lines with a redshift larger than that of the selected QSO. One of these objects is SDSS J0013+1523 ( $z_{\mathrm{QSO}}=0.120$; $\left.z_{\mathrm{s}}=0.641\right)$ as described in Courbin et al. (2011), where $z_{\mathrm{s}}$ is the redshift of the lensed background source. The three new objects studied in this paper are SDSS J0827+5224 ( $z_{\mathrm{QSO}}=0.293$; $\left.z_{\mathrm{s}}=0.412\right), \operatorname{SDSS~J0919+2720}\left(z_{\mathrm{QSO}}=0.209 ; z_{\mathrm{s}}=0.558\right)$, and SDSS J1005+4016 $\left(z_{\mathrm{QSO}}=0.230 ; z_{\mathrm{s}}=0.441\right)$. Figure 1 shows these three SDSS spectra, where the emission lines of the background object are marked in red.

Note that the present work is a pilot study aimed at discovering some of the objects with fairly low lens-source contrast and with as many background emission lines as possible. In doing this, we highly bias the "sample", both in redshift and in lens mass. In particular, the range of source redshifts is restricted to $0.4<z_{\mathrm{s}}<0.7$ in order to see four lines simultaneously. The selection function within this range is complicated due to the sky emission lines in the red part of the spectrum and to the iron lines in the foreground QSO. A detailed analysis of the selection window of our technique is well beyond the scope of this paper and depends on the adopted trade-off between the desired number of emission lines detected in the background sources and their detection level, i.e., the contrast between the source and the QSO. In addition, this contrast depends strongly on the relative redshifts of the QSO lens and the source.

\section{Keck spectroscopy and imaging}

We obtained spectroscopy of all three QSO lenses on UT 2010 March 12 with the dual-beam Low Resolution Imaging Spectrometer, LRIS (Oke et al. 1995; Rockosi et al. 2010), on the Keck I telescope. Two dithered exposures of each source were obtained. The night was photometric, with seeing of $\sim 1$ '. 2 . The observations used the 1 '. 5 wide longslit, the $5600 \AA$ dichroic, the $400 \ell \mathrm{mm}^{-1}$ grating on the red arm of the spectrograph (blazed at $8500 \AA$; spectral resolution $R \equiv \lambda / \Delta \lambda \approx 700$ for objects filling the slit), and the $600 \ell \mathrm{mm}^{-1}$ grism on the blue arm of the spectrograph (blazed at $4000 \AA ; R \approx 1000$ ). This configuration allows simultaneous coverage of essentially the entire optical window.

We observed SDSS J0827+5224 for a total integration time of $600 \mathrm{~s}$ at position angle $\mathrm{PA}=+34.1^{\circ}$. We observed SDSS $0919+2720$ for a total integration time of $900 \mathrm{~s}$ at PA = $+60.0^{\circ}$, and we observed SDSS $1005+4016$ for a total integration time of $1200 \mathrm{~s}$ at $\mathrm{PA}=-10.0^{\circ}$. Note that LRIS has an atmospheric dispersion corrector, so there are no issues with light lost due to observing at non-parallactic angles. The data were processed using standard procedures, and flux calibrated using archival observations of standard stars from Massey \& Gronwall (1990). The reduced spectra are presented in Fig. 2.

The imaging observations were obtained on the night of UT 2010 May 17, at the 10-m Keck I telescope in variable conditions. No flux calibration was obtained. Each object was observed in the $B$ and $R$ filters, with a pixel scale of $0.135^{\prime \prime}$. The exposure times, seeing, observational setup and airmass for all Keck observations are summarized in Table 1.

\section{Spectral principal component analysis}

The spectral principal component analysis (PCA) procedure developed by Boroson \& Lauer (2010) can be used to model any QSO spectrum. This technique uses spectra of a large, representative sample of objects to generate eigenspectra, which can be fit to a given spectrum to get weights. A linear combination of these eigenspectra can reproduce all the features of the input spectrum to within the noise of the observation, given a sufficiently large set of eigenspectra constructed from spectra with high signal-to-noise. However, features that are not properly part of the object spectrum will not be represented in the eigenspectra, and so will not appear in the reconstruction. Thus, the difference between the observed spectrum and the reconstructed model will show residual features due to a superposed object at a different redshift. We used this technique as an alternative method to view the lines from the lensed objects relative to the noise and to search for weaker lines from the lensed objects. This worked well for the SDSS spectra where we had a large number of QSOs with which to create the eigenspectra. An example is shown in Fig. 3, where the spectrum of SDSS J0827+5224 is modeled and subtracted, showing the residual lines from the lensed galaxy in the bottom panel. We did not detect any additional features from the lensed galaxies by this technique, but we plan to explore the use of this procedure to search for additional examples of objects lensed by QSOs within the SDSS archive. The main advantage of this approach over other techniques is that the many faint iron features present in the spectrum of the foreground QSO are well removed, hence avoiding the misidentification of emission lines from the background object with foreground iron emission. When applied to our Keck spectra, the PCA analysis shows prominent emission lines from the lensed sources, well above the noise level (Fig. 4). We plan to use this technique to find objects with high source/lens contrast, allowing us to expand our sample of these difficult to identify systems.

As we used this technique only to isolate features arising in the lensed object in previously discovered systems, we leave a complete analysis of its potential sensitivity to our future survey. However, we note that Boroson \& Lauer (2010) show that a spectral PCA model is able to reproduce the intrinsic spectrum with much greater fidelity than the input data typically do; thus, the significance of a feature detected in this way generally depends only on the signal-to-noise of the input spectrum, not on the quality of the model.

\section{Evidence for strong lensing from the Keck data}

The background emission lines detected in the SDSS spectra of our three QSOs are confirmed with the Keck spectra. With the longer wavelength coverage of the LRIS spectrograph, additional lines are even detected, like the strong $\mathrm{H} \alpha$ emission of a galaxy in the background of SDSS J1005+4016. 
F. Courbin et al.: Strong gravitational lensing by QSOs
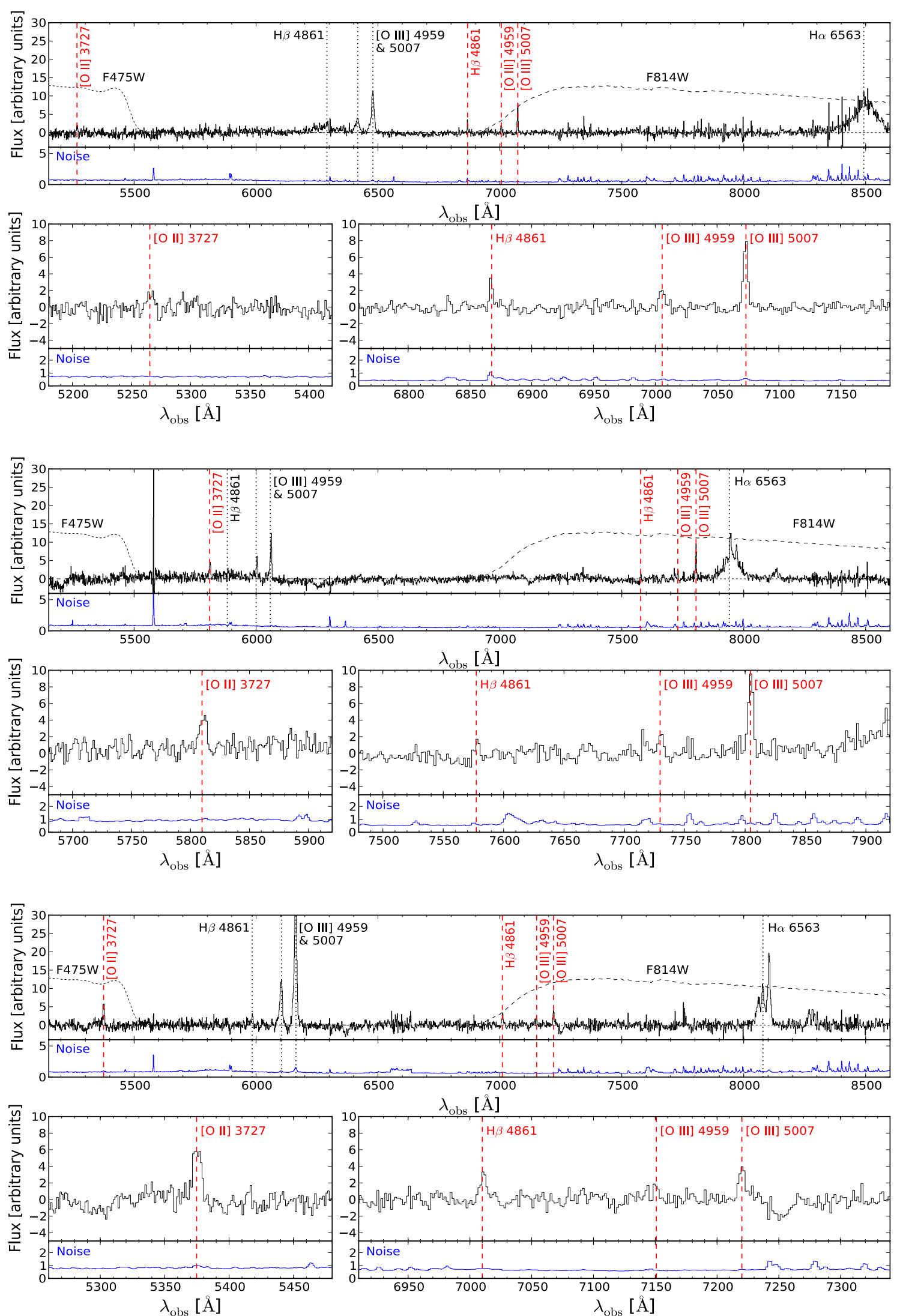

Fig. 1. SDSS spectra of the three new QSO lenses. From top to bottom are SDSS J0827+5224, SDSS J0919+2720, and SDSS J1005+4016. The emission lines of the foreground QSOs are indicated with black vertical lines (dotted) and the emission lines of the background objects are shown with red vertical lines (dashed). All three QSOs have four significant background emission lines. All spectra are shown in the observed frame. The HST filter curves are also displayed. 

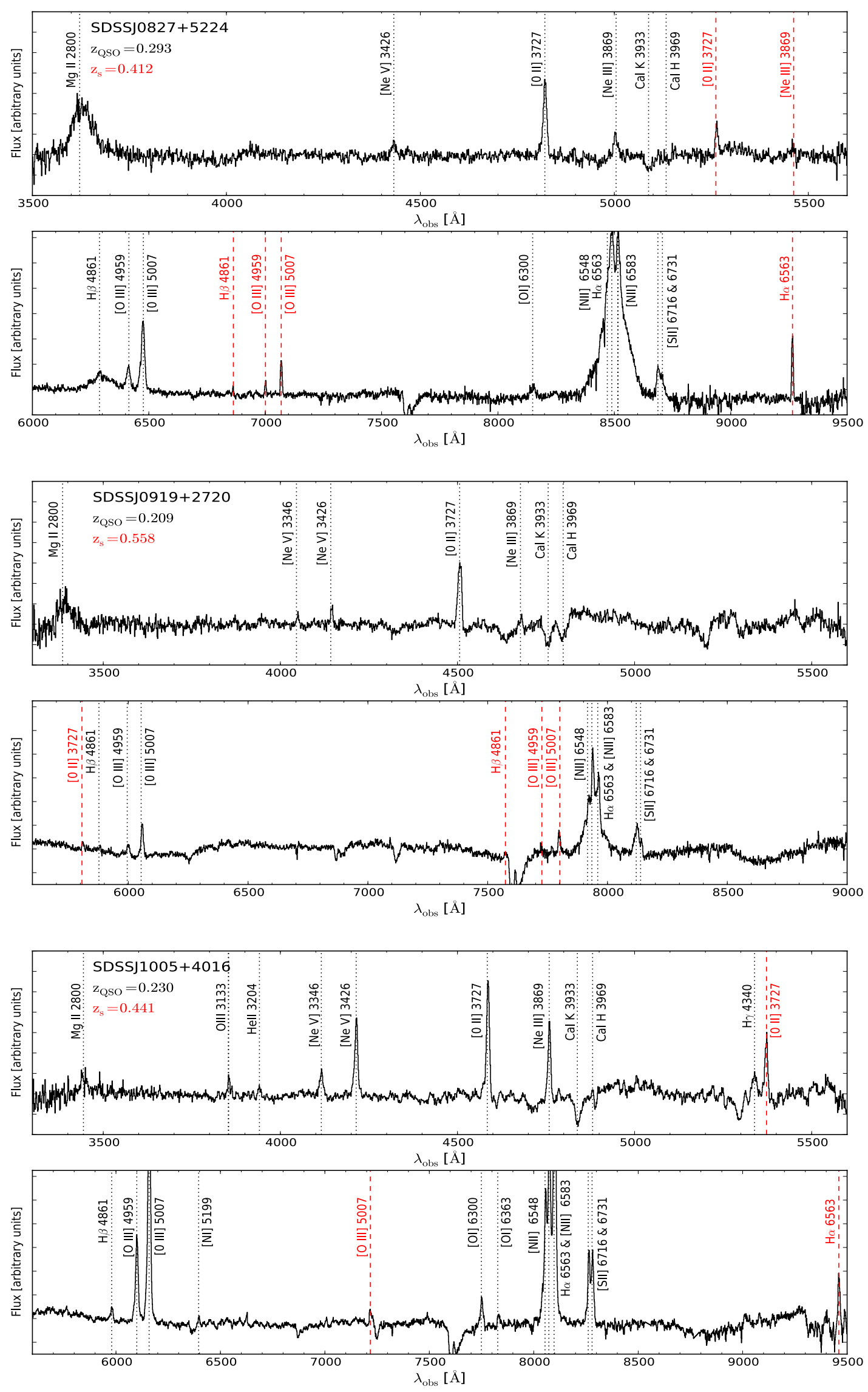

Fig. 2. Keck/LRIS spectra of the three new QSO lenses. From top to bottom are SDSS J0827+5224, SDSS J0919+2720, and SDSS J1005+4016. The emission lines are labelled as in Fig. 1. For each object the two panels correspond to the spectra obtained in the blue and red channels of the LRIS spectrograph. All spectra are shown in the observed frame. They have not been corrected for telluric absorption. 
Table 1. Summary of the Keck/LRIS observations.

\begin{tabular}{lrccc}
\hline \hline Object & $\begin{array}{r}\text { Exp. time } \\
(\mathrm{s})\end{array}$ & Filter & $\begin{array}{c}\text { Seeing } \\
\left({ }^{\prime \prime}\right)\end{array}$ \\
\hline SDSS J0827+5224 & 600 & $B$ & 0.81 & 1.39 \\
& 600 & $R$ & 0.90 & 1.39 \\
& 600 & G400 red & 1.20 & 1.19 \\
SDSS J0919+2720 & 600 & G600 blue & 1.20 & 1.19 \\
& 600 & $B$ & 0.75 & 1.13 \\
& 600 & $R$ & 0.75 & 1.13 \\
SDSS J1005+4016 & 900 & G400 red & 1.20 & 1.01 \\
& 900 & G600 blue & 1.20 & 1.01 \\
& 600 & $B$ & 0.75 & 1.12 \\
& 600 & $R$ & 0.70 & 1.12 \\
& 1200 & G400 red & 1.20 & 1.07 \\
& 1240 & G600 blue & 1.20 & 1.07 \\
\hline
\end{tabular}

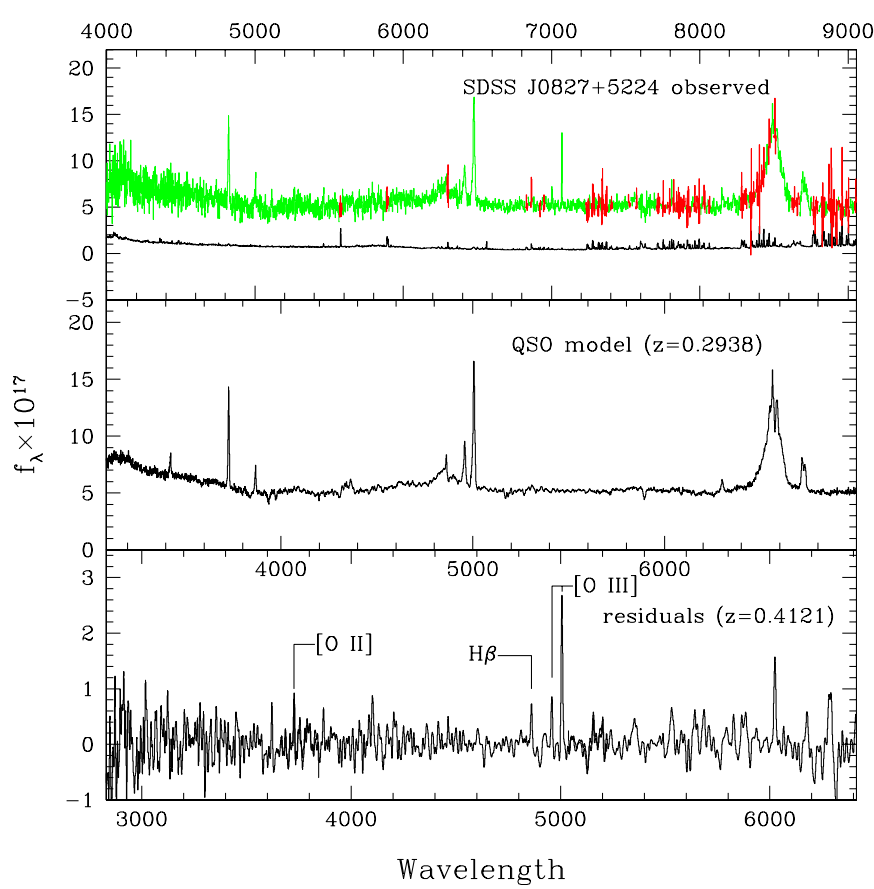

Fig. 3. Example of a spectral principal component analysis of a SDSS spectrum. Top: SDSS spectrum of SDSS J0827+5224 along with the $1 \sigma$ errors (black). The spectrum is shown in the observed frame, labeled on the upper axis. The red points are those flagged as bad in the SDSS archive, typically because of poor sky subtraction. Middle: model of this spectrum, computed using the 43 most significant eigenspectra derived from 1000 high signal-to-noise spectra from the SDSS archive. The model is plotted against rest wavelength of the QSO, labeled below the lower boundary of this panel. Bottom: subtraction of the model spectrum from the observed one, smoothed with a Gaussian having a full width at half maximum FWHM $=200 \mathrm{~km} \mathrm{~s}^{-1}$. The [OII], [OIII], and $\mathrm{H} \beta$ emission lines from the lensed object are labeled. This difference spectrum is plotted against rest wavelength in the frame of the lensed object. The significant peaks that are not labeled are all associated with regions of poor sky subtraction.

There is therefore little doubt that all three targets consist of a low redshift QSO almost perfectly aligned with a background emission line galaxy. Whether these background galaxies are strongly lensed or not depends on the surface mass density of the foreground QSO host galaxies.

We use our Keck optical images to look for lensed multiple images or arcs within $3^{\prime \prime}$ of the QSOs. Due to the brightness of the QSOs this is a challenging task with the spatial resolution of
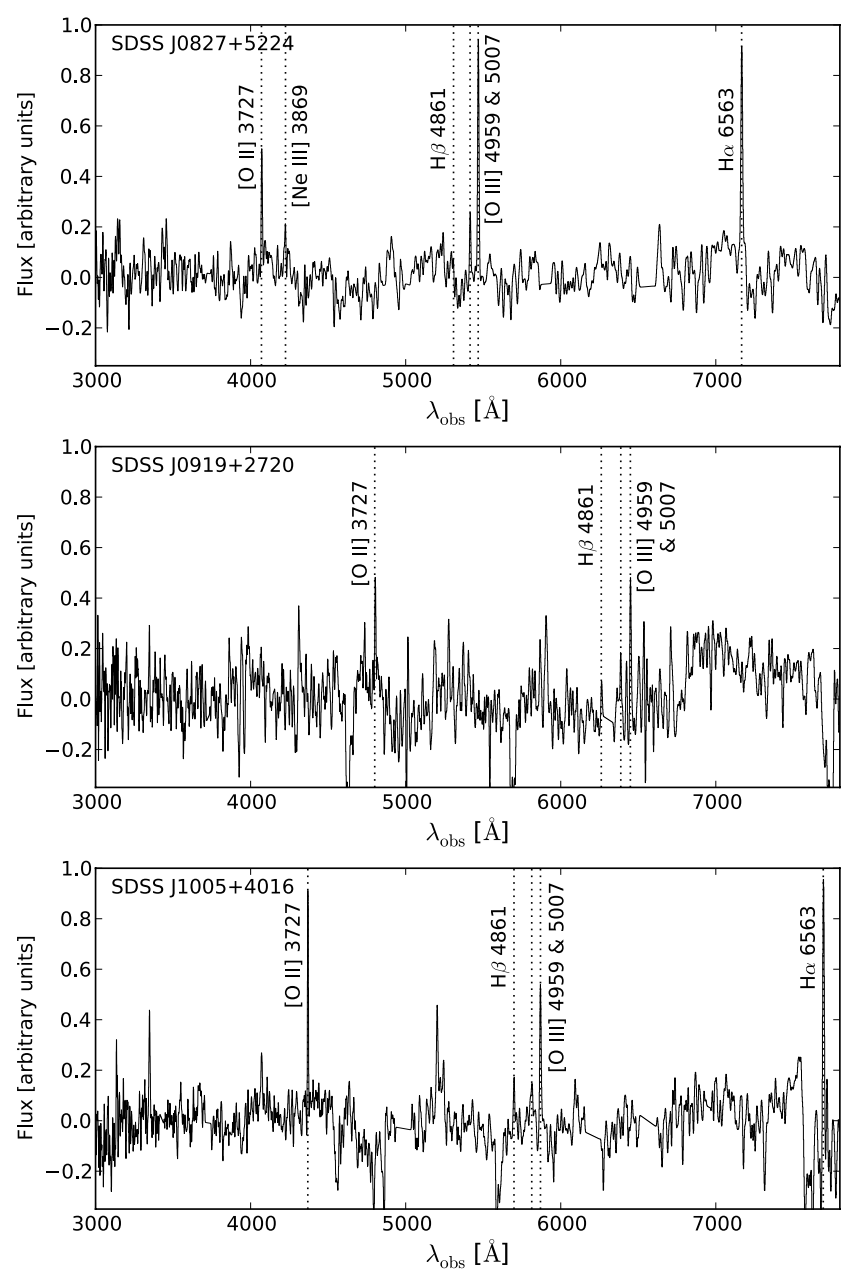

Fig. 4. PCA analysis of the Keck I spectra, shown in the observed frame. Between 3 and 6 of the emission lines of the background source are well detected depending on the object.

ground-based data. Image "deconvolution" is required. We apply the MCS deconvolution software (Magain et al. 1998) to the data of SDSS J0919+2720 and SDSS J1005+4016. No PSF star is available in the field of view of SDSS J0827+5224, so no reliable deconvolution is possible for that object. The images are shown in Figs. 5-7. When the deconvolution process is possible, the achieved resolution is FWHM $=0.135^{\prime \prime}$. Although only the HST images presented in Sect. 6 allow to us to draw firm conclusions about all three objects, we find it interesting to briefly describe how the Keck and SDSS data alone already give convincing clues that these are strong lenses.

\subsection{SDSS J0827+5224}

No image deconvolution is possible for this object. The images obtained in the two optical bands (Fig. 5) show no trace of any background object, in spite of the emission lines seen in the SDSS spectrum. These lines become striking in our Keck spectrum. This may be due to photometric variations of the source between the SDSS and the Keck observations or maybe due to aperture effects. Indeed, the $1.5^{\prime \prime}$ slit width of LRIS is twice as narrow as the $3^{\prime \prime}$ fiber of the SDSS spectrograph. The increased brightness of the background emission lines in the Keck spectrum, as compared with the SDSS spectrum, therefore suggests that the emitting object must be very close to the foreground 


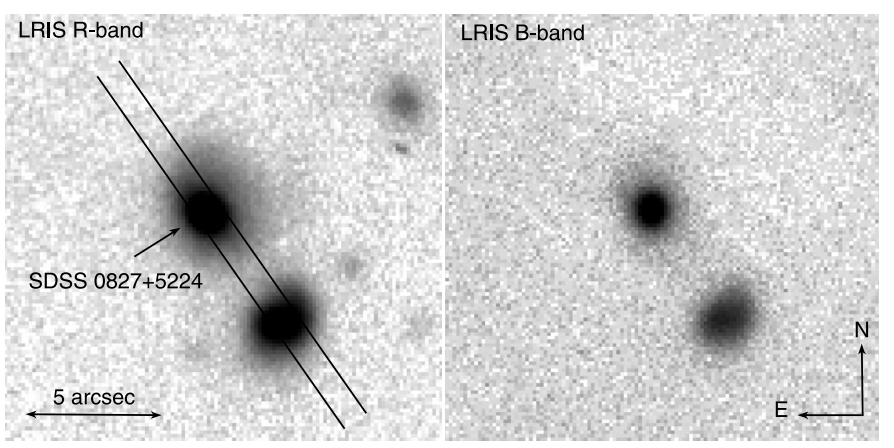

Fig. 5. Keck/LRIS images of SDSS J0827+5224 $(z=0.293)$ in the $R$ and $B$ bands. The $1.5^{\prime \prime}$ slit of the LRIS spectrograph is shown, oriented with $\mathrm{PA}=+30^{\circ}$.

QSO on the plane of the sky, too close to the QSO to be seen in our ground-based images.

From the Keck and SDSS data alone we conclude that we are in presence of two objects aligned with an accuracy of $0.75^{\prime \prime}$ (half the LRIS slit width) but the background object is not spatially resolved in the Keck images.

We note that the second object in the LRIS slit is a normal galaxy at the redshift of the QSO.

\subsection{SDSS J0919+2720}

Several PSF stars are available near SDSS J0919+2720. The deconvolved images (Fig. 6) reveal a complex system. Two objects are seen in the central arcsecond. One is extended and dominates the total flux in the $R$ filter ("lens" in Fig. 6). The other is compatible with being a very narrow blend of two point sources that dominates the flux in the $B$ filter ("A" in Fig. 6). The extended object is located at the center of a ring-like structure that makes a good candidate for a full Einstein ring. A plausible explanation is that the lensing galaxy is the red extended object, i.e., a low redshift early-type galaxy and that the point-like object is a QSO. The LRIS slit contains both objects. The integrated spectrum shows only two sets of lines at two different redshifts, not three. The QSO and the galaxy in Fig. 6 must therefore be at the same redshift.

Contrary to SDSS J0827+5224, the background emission lines are seen stronger in the SDSS spectrum with respect to the QSO lines than they do in the Keck spectrum. This is well explained by the slit orientation of LRIS, that catches most of the light of the putative lens and QSO, but which misses most of the flux from the ring-like structure. The SDSS fiber fully encompasses the ring, hence producing stronger lines.

We conclude that we see an early-type galaxy lensing a background source as a full Einstein ring, and that the lensing galaxy has a nearby QSO companion at the same redshift. It is not clear at this points whether the QSO has its own host galaxy or whether its host galaxy is the one at the center of the Einstein ring. Although the QSO is brighter than the galaxy, we expect it to be much less massive and to contribute only as a perturber to the main lensing potential well. Structures are seen in the ring (B and $\mathrm{C}$ in Fig. 6). It is not clear whether they are associated with the lensed source or whether they are unrelated objects seen in projection along the line of sight.
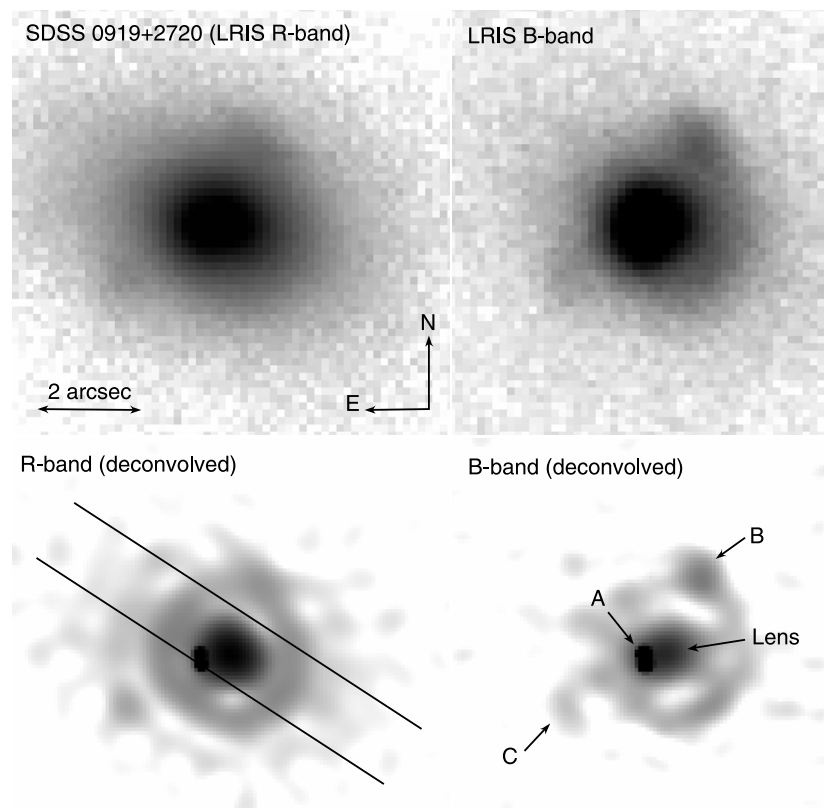

Fig. 6. Top: Keck/LRIS images of SDSS J0919+2720 $(z=0.209)$ in the $R$ and $B$ bands. Bottom: deconvolution of the two images unveiling a ring-like structure and a narrow pair of bright point sources, labelled A, that dominates the total flux in the blue. In contrast, the object in the center of the ring, i.e., the putative lensing object, is red. Two other objects are seen about the lens, labelled B and C. The $1.5^{\prime \prime}$ slit of the LRIS spectrograph is shown, and oriented with $\mathrm{PA}=+60^{\circ}$.

\subsection{SDSS J1005+4016}

The Keck images of SDSS J1005+4016 show a face-on spiral galaxy with a bright nucleus. Image deconvolution reveals a double nucleus, as shown in Fig. 7. One of the two "nuclei" is compatible with being a point source (QSO) superposed with an extended component, i.e., the nucleus of the spiral galaxy. The other, located about 1 " away from the QSO is elongated and not at the center of the spiral. If it is lensed by the QSO+bulge of the galaxy, it does not show any counter image symmetric about the QSO. This object is either not lensed or forms a partial Einstein ring. The HST images presented below demonstrate that the latter hypothesis is correct.

\section{Confirmation with HST/WFC3 imaging}

Our Keck images and spectra show strong evidence for lensing. However a detailed analysis of the lensed images and of the QSO host galaxy requires high resolution space images. We have obtained such HST data (program GO\#12233), with the Wide Field Camera 3 (WFC3) and the UVIS detector.

\subsection{Observations}

For each of the three objects, as well as for SDSS J0013+1523, previously published in Courbin et al. (2010), we took six dithered exposures in the $F 814 \mathrm{~W}$ filter and six exposures in the $F 475 W$ filter. These observations correspond to one full orbit per filter and per object.

In addition to the QSO observations, we observed the open cluster NGC 136 in order to build a high signal-to-noise model of the PSF at the position of the QSO. This cluster contains stars with colors similar to the QSOs and well sample the field of 

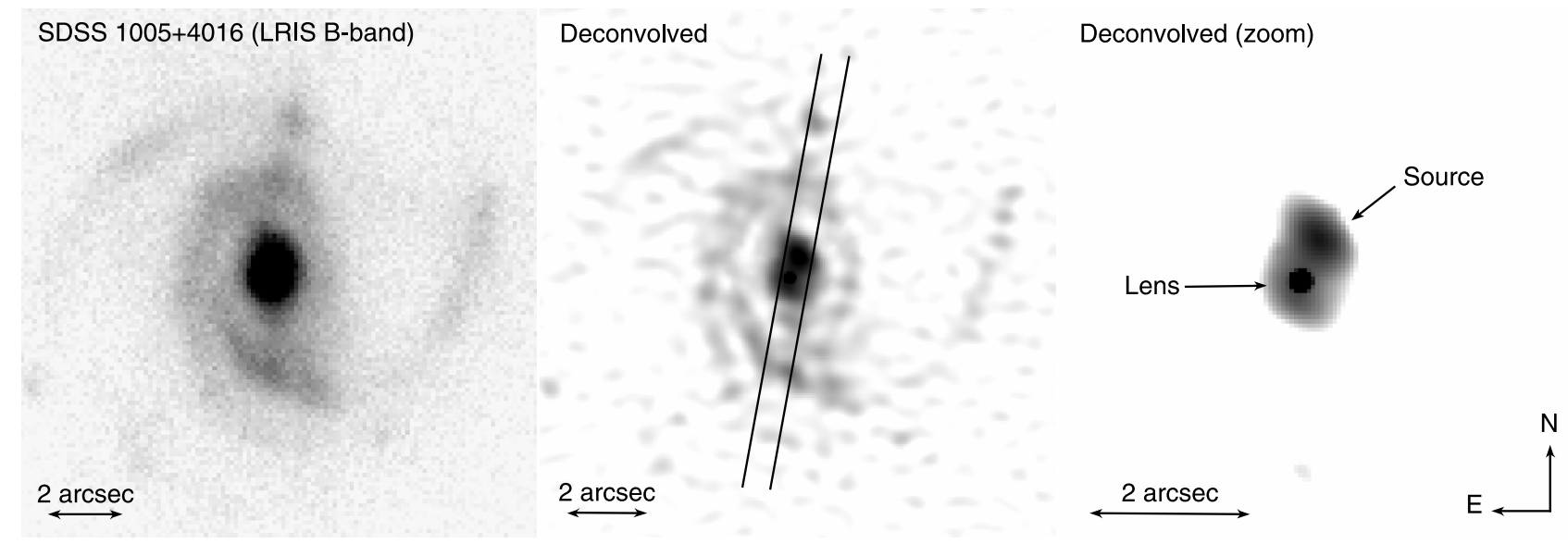

Fig. 7. Left: Keck/LRIS image of SDSS J1005+4016 $(z=0.230)$ obtained in the $B$-band showing the face-on spiral QSO host galaxy. Middle: deconvolved image showing that the nucleus of the spiral is double. The $1.5^{\prime \prime}$ slit of LRIS is shown in overlay, with PA $=-10^{\circ}$. Right: zoom of the center of the deconvolved image showing the bulge of the foreground spiral and a second object $\sim 1^{\prime \prime}$ to the NW.

view, while still avoiding heavy crowding. The brightness of the stars in this cluster also allow to apply exposure times that avoid strong saturation, even with the overhead times of WFC3 due to transfer of data frames in the camera buffer. NGC 136 was observed for one full orbit, using three long exposures (350 s) and one short exposure $(60 \mathrm{~s})$ for each filter.

\subsection{Subtraction of the QSO and its host galaxy}

We carry out a two dimensional decomposition of the data using the Galfit software (Peng et al. 2002, 2010), which is sufficient to identify which structures belong to the QSO+host and which can be associated with the lensed source. For this purpose we use the pipeline-drizzled images provided by STScI.

For each object, we first build a PSF in the two filters using the observations of NGC 136. This is done by building a color-magnitude diagram for the cluster and selecting stars with colors similar to our QSOs. The stars are also required to span a range of luminosities allowing to model both the core and the wings of the PSF.

We then fit a two-dimensional QSO+galaxy model convolved with the PSF and subtract it. The specific choice of the model is not important for the current application, as we only want to subtract as much light as possible from the foreground object to unveil the background source. However, the best fits are obtained in all cases by using a combination of one PSF plus one or two Sersic profiles. The results of this analysis is displayed in Fig. 8.

\section{Results}

The HST images immediately confirm the lensing nature of SDSS J0919+2720, which displays a prominent blue Einstein ring surrounding two bright objects. One of them is a pointsource, i.e., the foreground QSO, and the second, at the very center of the Einstein ring, is an early-type galaxy. The data reveals a dark extended structure centered on the QSO as well as a dark lane going through the galaxy. It is therefore likely that the lensing object is in fact a QSO+galaxy merger at $z=0.210$ with significant amount of dust.

The second striking object is SDSS J1005+4016. The elongated object seen in the deconvolved Keck images is beautifully confirmed as a partial lensed ring. A close look at the residual image after subtraction of the lensing QSO reveals a small object about $0.1^{\prime \prime}$ to the South-East of the QSO. This object is either produced by dusty structures in the nucleus of the spiral or a counter image of the arc. Detailed lensing models will tell whether it is a counter image of the arc of if we are observing a "naked cusp" arc.

SDSS J0827+5224 is also confirmed as a lens from the HST images. Two slightly extended sources are seen on each side of the central QSO after proper PSF subtraction (shown with arrows in Fig. 8). These objects are seen both in the F814W and in the F475W images, but the contrast with the QSO makes them most visible in the bluer $F 475 \mathrm{~W}$ filter. As suspected from the relative brightness of the background emission lines seen in the SDSS and Keck spectra, the two lensed images of the source are very close to the foreground QSO.

Finally, SDSS J0013+1523 is a more difficult system to confirm as a gravitational lens. Courbin et al. (2010) detected two point-like sources symmetrical about the foreground QSO, from near-IR adaptive optics images. The fainter of these sources is not visible in the optical HST images, either because it is heavily obscured by dust or because it was an artifact in the Keck adaptive optics images. The brighter of the two sources, labelled A in Fig. 8, is confirmed. It shows an extended arc-like structure to the North that could consist of a partial Einstein ring though this structure is faint and affected by the foreground QSO host galaxy. Therefore, with the present data set, we cannot conclude with certainty that SDSS J0013+1523 is a genuine lens.

Basic measurements are given for all four systems in Table 2. The mass estimates are derived from a Singular Isothermal Sphere (SIS) lens model. In Table 2 the error bar on the total mass, $M\left(<R_{\mathrm{E}}\right)$, is of the order of $15 \%$. This is due to the arbitrary choice of an isothermal mass profile to determine the lens total mass, and to the fact that we approximate $R_{\mathrm{E}}$ by assuming it is equal to the size of the Einstein rings seen in the HST images. The error is then propagated in the calculation of the line of sight velocity dispersion. The latter assumes spherical symmetry for the lens. The model assumes that the counter-image of the arc in SDSS J1005+4016 is real, i.e., that the arc is not a naked-cusp. For SDSS J0013+1523, using a single image of the source to constrain the model leads to an upper limit for the mass of the QSO lens. 

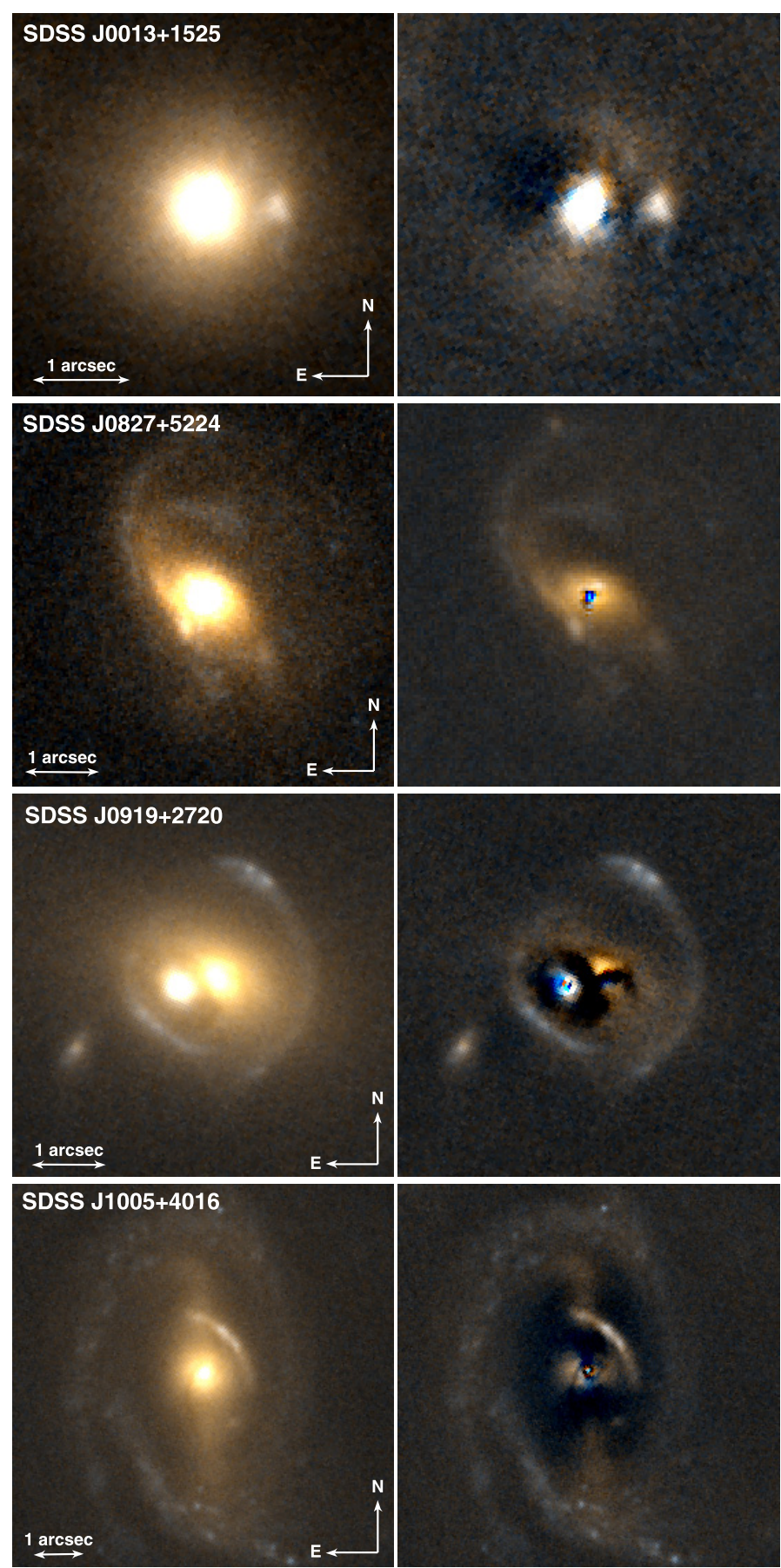

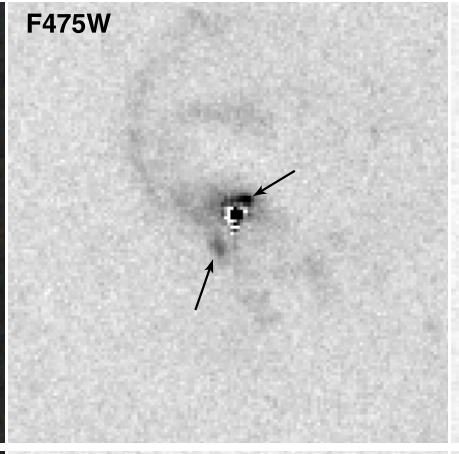

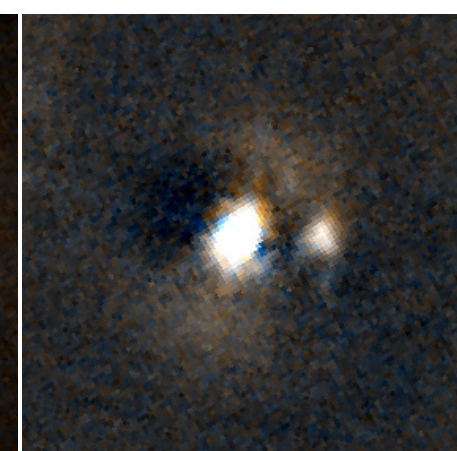

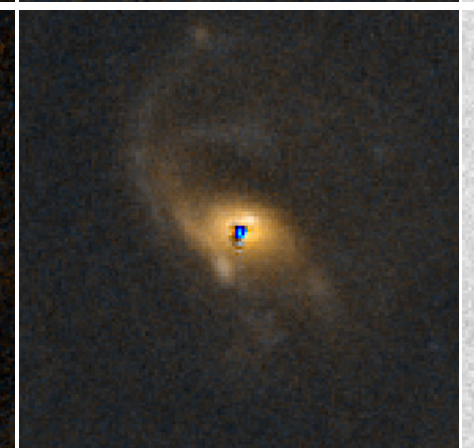

\section{F475W}

F475W

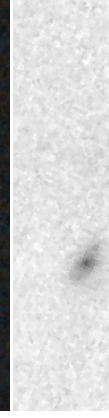

F475W

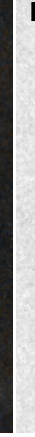

\section{F475W}

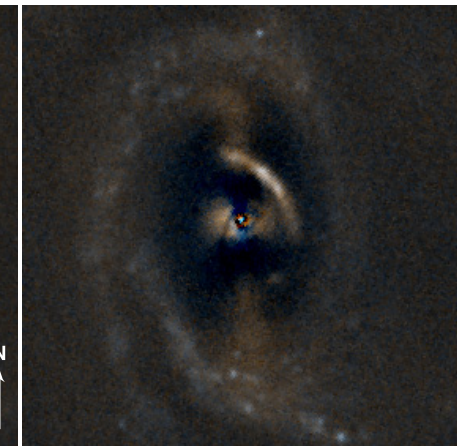

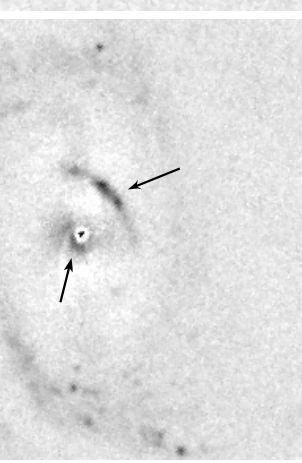

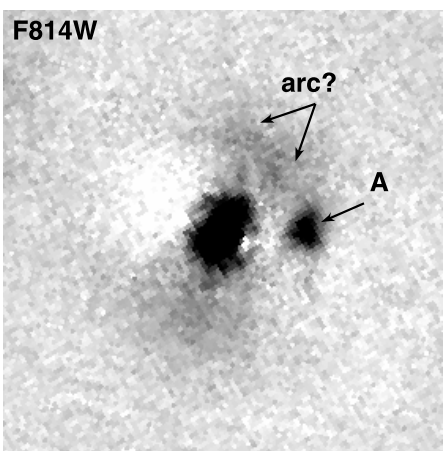

F814W

F814W

F814W

Fig. 8. HST/WFC3 images of our lensing QSOs. In each row, from left to right, we show (i) a color composite of the system, (ii) a color composite after subtraction of the QSO and of the central parts of its host galaxy, (iii) the F475W image after subtraction of the QSO and its host, (iv) the F814W image after subtraction of the QSO and its host. The arrows and labels indicate the lensed structures described in Sect. 7. Note that SDSS J0013+1523 was already presented in Courbin et al. (2010) but the present HST images do not clearly support the lensing nature of this object.

\section{Conclusion}

Our combined Keck spectroscopic and imaging dataset, as well as the HST/WFC3 optical images, allow us to confirm three new QSOs acting as strong lenses of background galaxies. Two of them, SDSS J0827+5224 and SDSS J1005+4016, have faceon host galaxies and background lensed images that probe the very central part of the lensing potential. Detailed lens modeling combined with dynamical information on the host galaxies (rotation curve and central velocity dispersion) may therefore be very effective in breaking the bulge-halo degeneracy. The third object, SDSS J0919+2720, is peculiar and consists of a QSO+galaxy merger. Gravitational lensing will offer an opportunity to model accurately the mass distribution in the pair and possibly to discriminate between the mass of the galaxy and that of the companion QSO. The lensing models needed to study these three unique active galaxies is beyond the scope of the present discovery paper and will be detailed in future work.

The three objects presented here are the ones with the strongest background emission lines in SDSS DR7. Fainter and 
Table 2. Basic measurements for each object, including QSO and source redshifts, absolute magnitude of the QSO and of its host galaxy after subtraction of the QSO light.

\begin{tabular}{lcccccccccc}
\hline \hline Name & $z_{\mathrm{QSO}}$ & $z_{\mathrm{s}}$ & $\begin{array}{c}F 475 W \\
\text { QSO }\end{array}$ & $\begin{array}{c}F 814 W \\
\text { QSO }\end{array}$ & $\begin{array}{c}F 475 W \\
\text { Host }\end{array}$ & $\begin{array}{c}F 814 W \\
\text { Host }\end{array}$ & $\begin{array}{c}R_{\mathrm{E}} \\
\left({ }^{\prime \prime}\right)\end{array}$ & $\begin{array}{c}M\left(<R_{\mathrm{E}}\right) \\
\left(10^{10} M_{\odot}\right)\end{array}$ & $\begin{array}{c}\sigma_{v, 3 \mathrm{D}} \\
\left(\mathrm{km} \mathrm{s}^{-1}\right)\end{array}$ & $\begin{array}{c}\sigma_{v, \mathrm{LOS}} \\
\left(\mathrm{km} \mathrm{s}^{-1}\right)\end{array}$ \\
\hline SDSS J0013+1525 & 0.120 & 0.641 & -18.46 & -18.75 & -18.75 & -20.15 & $<0.74$ & $<3.7$ & $<180.9$ & $<192$ \\
SDSS J0827+5224 & 0.293 & 0.412 & -19.95 & -20.89 & -19.05 & -19.61 & 0.31 & $3.8 \pm 0.6$ & $201_{-9}^{+5}$ & $213_{-9}^{+5}$ \\
SDSS J0919+2720 & 0.209 & 0.558 & -19.04 & -19.46 & -19.77 & -21.80 & 1.22 & $21.4 \pm 6.7$ & $268_{-11}^{+9}$ & $284_{-11}^{+9}$ \\
SDSS J1005+4016 & 0.230 & 0.441 & -17.29 & -18.86 & -19.06 & -21.15 & 0.61 & $7.5 \pm 1.1$ & $217_{-9}^{+7}$ & $230_{-9}^{+7}$ \\
\hline
\end{tabular}

Notes. The measurements are given in the observed frame, in the Einstein radius. No k-correction is applied. (8) Einstein radius (9) total mass in the Einstein radius $(10,11)$ 3D stellar velocity dispersion and projected line of sight velocity dispersion, as calculated from our lensing model, assuming a circular, isotropic, and isothermal profile.

more numerous lines may be detected with cleaner QSO subtraction using the Spectral Principle Component Analysis technique. There is therefore hope to build a statistically significant sample of "QSO lenses" to study how the properties of QSOs and their host galaxies change with host mass and possibly with redshift.

Acknowledgements. This study is supported by the Swiss National Science Foundation (SNSF). S.G.D. and A.A.M. acknowledge a partial support from the NASA grant HST-GO-12233.01-A, the NSF grant AST-0909182, and the Ajax Foundation. The work of D. Stern was carried out at Jet Propulsion Laboratory, California Institute of Technology, under a contract with NASA. D. Sluse acknowledges partial support from the German Virtual Observatory and from the Deutsche Forschungsgemeinschaft, reference SL172/1-1.

This work makes use of the data collected by the SDSS collaboration and released in DR7. Funding for the SDSS and SDSS-II has been provided by the Alfred P. Sloan Foundation, the Participating Institutions, the National Science Foundation, the US Department of Energy, the National Aeronautics and Space Administration, the Japanese Monbukagakusho, the Max Planck Society, and the Higher Education Funding Council for England. The SDSS Web Site is http: // www. sdss. org/.

The SDSS is managed by the Astrophysical Research Consortium for the Participating Institutions. The Participating Institutions are the American Museum of Natural History, Astrophysical Institute Potsdam, University of Basel, University of Cambridge, Case Western Reserve University, University of Chicago, Drexel University, Fermilab, the Institute for Advanced Study, the Japan Participation Group, Johns Hopkins University, the Joint Institute for Nuclear Astrophysics, the Kavli Institute for Particle Astrophysics and Cosmology, the Korean Scientist Group, the Chinese Academy of Sciences (LAMOST), Los Alamos National Laboratory, the Max-Planck-Institute for Astronomy (MPIA), the Max-Planck-Institute for Astrophysics (MPA), New Mexico State University, Ohio State University, University of Pittsburgh, University of Portsmouth, Princeton University, the United States Naval Observatory, and the University of Washington.

\section{References}

Abazajian, K. N., Adelman-McCarthy, J. K., Agüeros, M. A., et al. 2009, ApJS, 182,543

Auger, M. W., Treu, T., Bolton, A. S., et al. 2010, ApJ, 724, 511

Barnabè, M., Czoske, O., Koopmans, L. V. E., et al. 2009, MNRAS, 399, 21

Biggs, A. D., Browne, I. W. A., Jackson, N. J., et al. 2004, MNRAS, 350, 949

Bolton, A. S., Burles, S., Koopmans, L. V. E., et al. 2008, ApJ, 682, 964

Bonning, E. W., Shields, G. A., Salviander, S., \& McLure, R. J. 2005, ApJ, 626, 89

Boroson, T. A., \& Lauer, T. R. 2010, AJ, 140, 390

Chantry, V., Sluse, D., \& Magain, P. 2010, A\&A, 522, A95

Courbin, F., Tewes, M., Djorgovski, S. G., et al. 2010, A\&A, 516, L12

Courbin, F., Chantry, V., Revaz, Y., et al. 2011, A\&A, 536, A53

Faure, C., Kneib, J., Covone, G., et al. 2008, ApJS, 176, 19

Kaspi, S., Maoz, D., Netzer, H., et al. 2005, ApJ, 629, 61

Keeton, C. R., Gaudi, B. S., \& Petters, A. O. 2003, ApJ, 598, 138

Kochanek, C. S., \& Dalal, N. 2004, ApJ, 610, 69

Koopmans, L. V. E., Garrett, M. A., Blandford, R. D., et al. 2002, MNRAS, 334, 39

Magain, P., Courbin, F., \& Sohy, S. 1998, ApJ, 494, 472

Mao, S., \& Schneider, P. 1998, MNRAS, 295, 587

Massey, P., \& Gronwall, C. 1990, ApJ, 358, 344

Muñoz, J. A., Falco, E. E., Kochanek, C. S., et al. 1998, Ap\&SS, 263, 51

Oke, J. B., Cohen, J. G., Carr, M., et al. 1995, PASP, 107, 375

Peng, C. Y., Ho, L. C., Impey, C. D., \& Rix, H. 2002, AJ, 124, 266

Peng, C. Y., Ho, L. C., Impey, C. D., \& Rix, H. 2010, AJ, 139, 2097

Refsdal, S. 1964, MNRAS, 128, 307

Rockosi, C., Stover, R., Kibrick, R., et al. 2010, in SPIE Conf. Ser., 7735

Shen, J., Vanden Berk, D. E., Schneider, D. P., \& Hall, P. B. 2008, AJ, 135, 928

Suyu, S. H., Marshall, P. J., Auger, M. W., et al. 2010, ApJ, 711, 201

Vegetti, S., Koopmans, L. V. E., Bolton, A., Treu, T., \& Gavazzi, R. 2010, MNRAS, 408, 1969

Warren, S. J., Hewett, P. C., Lewis, G. F., et al. 1996, MNRAS, 278, 139

Yoo, J., Kochanek, C. S., Falco, E. E., \& McLeod, B. A. 2005, ApJ, 626, 51 